\title{
EL USO DE LA ANTONIMIA EN EL LIBRO I DE LOS EPIGRAMAS DE MARCIAL
}

\section{PEDRo JuAn GALÁN SÁNCHEZ}

Universidad de Extremadura

This paper analyses the use that Martial makes of antonymy in Book I of his Epigrams. The study reveals that this lexical resource stands out as a major stylistic device in Martial's epigrammatic work: almost $25 \%$ of the 118 epigrams in Book I resort to antonymy in the construction of their characteristic witty endings. The study goes on to show that the same pairs of antonyms - or very similar ones - can be found throughout the rest of Martial's Epigrams.

\section{Propósito}

El presente artículo tiene como objeto analizar el uso que Marcial hace del recurso léxico-estilístico de la antonimia. Limitaremos el análisis únicamente al libro I de los Epigramas. Se trata, en todo caso, de una cala lo suficientemente significativa como para que las conclusiones a las que lleguemos puedan ser aplicadas - con las debidas precauciones, como es natural al resto de la obra de Marcial. Por otro lado, analizaremos sólo los antónimos que aparecen en lo que tradicionalmente se ha denominado la punta epigramática, o agudeza final, porque es justamente ahí, en el último dístico o en el último verso del epigrama, donde el recurso de la antonimia alcanza una importancia estilística capital en la obra de Marcial.

La antonimia, si bien se mira, no es más que una variante de un procedimiento estilístico de mayor calado, la figura retórica de la antítesis ${ }^{1}$, que,

1 Como señala E. Rodón Binué, «La expresividad léxica en Marcial», en Actas del Sim- 
como es bien sabido, puede ser expresada mediante dos procedimientos básicos, a saber, o léxicos o gramaticales. En este trabajo nos centraremos exclusivamente en el análisis de los procedimientos léxicos antitéticos utilizados por Marcial.

Pues bien, desde el punto de vista léxico, el recurso habitual para la expresión de ideas o pensamientos antitéticos es el uso de antónimos, entendiendo por antonimia lo que tradicionalmente se entiende, es decir, la "contrariedad" u oposición de significados: frío / calor; alto / bajo; amor / odio; claro / oscuro; antes / después, etc. Ahora bien, la antonimia pone en juego diversos tipos de "contrariedades", o contraposiciones lógicas. Así, por ejemplo, la relación de antonimia que se establece entre los siguientes cuatro pares de palabras es diferente en cada caso: soltero / casado, caliente / frío, dar / recibir, preguntar / responder. Todos son antónimos, pero cada una de estas cuatro parejas es antonímica en función de un criterio diferente. La cuestión de las subdivisiones de la antonimia no es algo fácil de establecer, y por ello mismo el asunto no ha sido resuelto definitivamente por los especialistas en Semántica ${ }^{2}$. Por eso, a la hora de elaborar este trabajo nos ha parecido oportuno proponer nuestra propia subdivisión, a fin de disponer, sobre todo, de un instrumento claro con el que intentar alcanzar un mejor entendimiento del asunto.

Pues bien, en nuestra opinión habría que distinguir dos grandes grupos de antónimos. En el primero estarían aquellos pares de palabras a los que se podría denominar "antónimos opuestos", es decir, aquellos que establecen entre sí una auténtica y radical contraposición; antónimos a los que, además, se les podría caracterizar como "insolidarios", en el sentido de que los dos vocablos que intervienen en la antonimia no se relacionan entre ellos de un

posio sobre Marco Valerio Marcial, poeta de Bílbilis y de Roma, Zaragoza, 1987, p. 299, dos de los principios fundamentales de la técnica poética de Marcial son la "recurrencia" y la "contraposición". Pues bien, es dentro del principio general de la "contraposición" donde hay que situar al procedimiento particular de la "antítesis". Para una relación de epigramas de Marcial en los que se recurre al uso de la antítesis, vid. J. Kruuse, "L'originalité artistique de Martial, son style, sa composition, sa technique”, Classica e Medievalia 4, 1941, p. 287, n. 3.

2 Para una visión panorámica - con abundantes referencias bibliográficas - de los diferentes estudios sobre el fenómeno lingüístico de la antonimia, en donde se pone de manifiesto la gran variedad metodológica y terminológica sobre el asunto, vid. M. Martínez Hernández, Semántica del griego antiguo, Madrid, 1997, pp. 289-306. 
modo positivo, sino que más bien sus significados, si vale la expresión, se repugnan entre sí: hablar/callar; caliente/frío. En el segundo grupo de antónimos estarían aquellos pares de palabras a los que se podría denominar "antónimos recíprocos", es decir, aquellos en los que los dos términos de la antonimia, más que enfrentarse o contraponerse entre sí, se correlacionan; y en ese sentido se les podría caracterizar como antónimos "solidarios", pues en este caso los dos términos de la antonimia se relacionan entre sí de un modo positivo y, por así decir, fluido y amistoso: comprar / vender, preguntar / responder'.

Cada uno de los dos grandes grupos de antónimos descritos ("opuestos" y "recíprocos") se podría dividir, a su vez, en otros dos subgrupos, lo que nos daría cuatro clases diferentes de antónimos. Concretamente, dentro de los "antónimos opuestos" habría que distinguir - siguiendo ahora la terminología tradicional - entre los "antónimos complementarios" y los "antónimos contrarios". Los "antónimos complementarios", o "contradictorios" responderían a la siguiente fórmula: "La afirmación de A implica necesariamente la negación de $\mathrm{B}$, y la negación de A implica necesariamente la afirmación de B". Esquemáticamente: $A>-B ;-A>B$. En última instancia, estaríamos ante el tipo de antonimia que responde al criterio de la "disyunción exclusiva", o, dicho de otro modo, aquella que se basa en el hecho de que un término es la negación del otro y viceversa ${ }^{3}$. Es la oposición que se establece, por ejemplo, entre casado / soltero. En efecto, "casado" implica necesariamente "no soltero"; y viceversa, "no casado" implica necesariamente "soltero" 4 . Por su parte, los antónimos "contrarios" responderían a esta otra fórmula: "La afirmación de A implica necesariamente la negación de B, pero la negación de A no implica necesariamente la afirmación

3 Se trata de un tipo de antonimia aceptada por la generalidad de los lingüistas. Cf. J. Lyons, Introducción en la lingüística teórica (vers. cast. R. Cerdà), Barcelona, 1971, pp. 4746; S. Gutiérrez Ordóñez, Introducción a la semántica funcional, Madrid, 1989, pp. 131-2; M. Justo Gil, Fundamentos del análisis semántico, Santiago de Compostela, 1990, pp. 42-3; R. Martin, «Logique et mécanisme de l'antonymie», Travaux de Linguistique et de Littérature 11, 1973, pp. 37-51. M. Iliescu, «Oppositions sémantiques. Antonymie linguistique et antonymie logique», Folia Linguistica 10, 1977, pp. 151-168.

4 Otras parejas de "antónimos complementarios" serían, por ejemplo, útil / inútil, vivo / muerto, macho / hembra, hablar / callar. Se trata de lo que la lógica clásica denomina opuestos "contradictorios": "si A es verdadero, B es falso; si A es falso, B es verdadero". 
de B". Esquemáticamente: $A>-B ;-A n o>B^{5}$. Ahora, un término no es ya la negación del otro, sino que nos encontramos más bien ante la contraposición o enfrentamiento de dos conceptos que se hallan en los dos extremos o los dos polos de una misma realidad conceptual. Se trataría, por así decir, de términos polarizados y mutuamente antagonistas, términos que responderían a la definición lógica de "incompatibilidad": caliente / frío, amar / odiar, ir / venir. En efecto, "caliente" implica necesariamente "no frío", pero "no caliente" no implica necesariamente "frío"; o bien, "amar" implica necesariamente "no odiar", pero "no amar" no implica necesariamente "odiar", etc. ${ }^{6}$

En cuanto al segundo gran grupo de antónimos, los "antónimos recíprocos", se podrían subdividir en "antónimos inversos" y "antónimos alternos". Los "antónimos inversos" obedecerían a la siguiente fórmula: "La existencia de A implica necesariamente la existencia de B, y la existencia de B implica

\footnotetext{
5 Obviamente quedan excluidas de la fórmula oposiciones del tipo rosa / camelia o caliente / tibio, cuya relación no es de antonimia, sino de cohiponimia (ya sea serial o gradual).

6 Otras parejas de antónimos contrarios serían, por ejemplo, afirmar / negar, ir / venir, meter / sacar, subir / bajar, traer / llevar', grande / pequeño', etc. Se trata, a grandes rasgos, de lo que la lógica clásica denomina opuestos "contrarios": "si A es verdadero, B es falso; si A es falso, B puede ser falso o puede ser verdadero". Muchos lingüistas únicamente consideran "antónimos contrarios" a los "antónimos graduales" (caliente / frío; alto / bajo). Nosotros incluimos aquí también los pares de palabras del tipo amor / odio, abrir / cerrar, ir / venir, etc., ya que se trata también de términos "polarizados y mutuamente antagonistas". De hecho, así es como lo entiende, por ejemplo, R. Martin, «Logique et mécanisme de l'antonymie», en Travaux de Linguistique et de Littérature 11, 1973, pp. 37-51, quien considera que este tipo de pares responden al «semantismo de la inversión», ya sea inversión de posición (superior / inferior), inversión de dirección (ir / venir, subir / bajar) o inversión de grado (caliente / frío). Por su parte, J. Lyons, Semántica (vers. española de R. Cerdà, Barcelona, 1980, pp. 263 y ss.) a una parte de estos antónimos, aquellos que tienen que ver con relaciones espaciales (ir / venir, arriba / abajo, derecha / izquierda, llegar / partir, etc.), los incluye dentro un grupo diferenciado de antónimos, a los que denomina "antónimos direccionales". Finalmente, H. Geckeler, «Antonymie und Wortart», en Integrale Linguistik. Festschrift für H. Gipper, Amsterdam, 1979, pp. 455-482, a los antónimos del tipo 'amar/odiar' los denomina "antónimos antitéticos", a los del tipo abrir / cerrar "antónimos reversibles" y a los del tipo ir / venir "antónimos contradireccionales". Nosotros, reconociendo que las distinciones de Geckeler son completamente pertinentes, a todo este conglomerado de antónimos los denominaremos en este trabajo, en aras de la simplificación, "antónimos contrarios", por cuanto todos ellos responden a la fórmula propuesta para tales antónimos: $A>-B ;-A$ no $>B$.
} 
necesariamente la existencia de $\mathrm{A} "$ ". Esquemáticamente: $A>B ; B>A$. En este caso estaríamos ante una relación de "reciprocidad necesaria" entre los dos antónimos: dar / recibir. En efecto, la existencia de alguien que "da" implica necesariamente - y de un modo simultáneo - la existencia de alguien que "recibe"; y a la inversa, la existencia de alguien que "recibe" implica necesariamente la existencia de alguien que "da". Por último, los "antónimos alternos" responderían a la fórmula siguiente: "La existencia de A no implica necesariamente la existencia de B, pero la existencia de B sí implica necesariamente la existencia de A". Esquemáticamente: $A$ no $>B ; B$ $>A$. En este caso estaríamos ante una relación de "reciprocidad potestativa", en la que, además, los dos términos que intervienen en la antonimia no son simultáneos, sino sucesivos o alternos: dar / devolver. En efecto, "dar" no implica necesariamente "devolver", pero "devolver" sí implica necesariamente que alguien "ha dado"".

Una vez establecida la existencia de estos cuatro tipos diferentes de antónimos, procederemos ya a analizar el uso que Marcial hace de la antonimia en el libro I de los Epigramas.

7 Para S. Gutiérrez Ordóñez, ob. cit., p. 133, la "inversión” no es, en sentido estricto, una relación antonímica. Sí lo es, en cambio, para J. Lyons, Introducción en la lingüística teórica (vers. española de R. Cerdà), Barcelona, 1971, pp. 481-2; para H. Geckeler, art. cit., pp. 455 y ss.; o para M. Justo Gil, ob. cit. p. 44.

8 Otras parejas de "antónimos inversos" serían, por ejemplo, las siguientes: marido / esposa, amo / esclavo, tío / sobrino, maestro / discípulo, padre / hijo, médico / paciente, vender / comprar, pagar / cobrar, prestar / deber, hablar / escuchar, enseñar / aprender.

9 Este tipo de "antónimos alternos" no es reconocido por muchos especialistas como antónimos propiamente dichos. Bastantes estudiosos del asunto ni siquiera los mencionan. Por su parte, J. Lyons, Introducción en la lingüística teórica, cit., p. 483, aunque advierte que este tipo de términos presenta una estrecha relación con los llamados "antónimos inversos", por estar «permutacionalmente relacionados del mismo modo que los términos inversos», no llega finalmente a concederles el estatuto de antónimos. Por su parte, H. Geckeler, art. cit., les da abiertamente tal estatuto, bajo el criterio de que este tipo de pares establecen una «relación antecedente-consecuente». Otras parejas de "antónimos alternos" serían, por ejemplo, preguntar / responder, ofrecer / aceptar, buscar / encontrar, saludar / devolver el saludo, escribir una carta / contestar a una carta, invitar / corresponder a una invitación, etc. Como señala B. García Hernández, «Estructuras léxicas en los Epigramas de Marcial», en Actas del Simposio sobre Marco Valerio Marcial, poeta de Bilbilis y de Roma, Zaragoza, 1987, p. 247, hay un prefijo latino singularmente adecuado para indicar este tipo de relación, $r e(d)$ : rescribo, reuoco, resaluto. 


\section{Antónimos “complementarios"}

El primer ejemplo de antónimos "complementarios" lo encontramos en el conocido epigrama en el que Marcial contrapone a la obscenidad o procacidad de su obra la honestidad de su vida y de su conducta personal:

lasciua est nobis pagina, uita proba ${ }^{10}$.

La maestría de Marcial deja, como siempre, la agudeza para el último verso del epigrama. Y su exquisitez formal le lleva a utilizar para la expresión del pensamiento una equilibrada estructura en quiasmo: lasciua pagina / uita proba (adjetivo-sustantivo / sustantivo-adjetivo), con uso de antónimos "complementarios" colocados en principio y final de verso y aplicados a la conocida dualidad "vida / obra" 1 .

Veamos un segundo ejemplo:

nolo uirum facili redemit qui sanguine famam hunc uolo, laudari qui sine morte potest ${ }^{12}$.

En esta ocasión estamos ante una estructura en la que los dos antónimos complementarios (nolo / uolo) aparecen ambos a principio de verso y, de nuevo, siguiendo una estructura quiástica: nolo uirum / hunc uolo (Verbo-O. Directo / O. Directo-Verbo). Pero no es ésta la única oposición antonímica que existe en el dístico. Hay, en efecto, una segunda expresión antonímica por la que se oponen los conceptos complementarios "muerto / vivo": facili sanguine / sine morte. El sentido, en efecto, del dístico es el siguiente: "no quiero un héroe muerto / quiero un héroe vivo"13.

10 Mart., Epigr. I 4. Esta misma oposición, bajo la forma castus / improbus, vuelve a aparecer en Epigr. III 86, en donde se trata de este mismo asunto.

${ }_{11}$ Acerca de la exquisitez formal de Marcial, v. J. Kruuse, «L'originalité artistique de Martial, son style, sa composition, sa technique», Classica et Medievalia, 4, 1941, pp. 248300 .

12 Mart., Epigr. I 8. La oposición nolo / uolo reaparece en la punta epigramática de los siguientes epigramas: Epigr. II 49; VIII 44; X 8; XI 27; XII 94; XIV 1; y V 83. En este último, el verso es el siguiente: uelle tuum nolo, Dindyme, nolle uolo, con la doble oposición uelle nolo / nolle uolo.

13 Por lo demás, esta oposición antonímica vivo / muerto, vivir / morir es muy del gusto de Marcial, como lo demuestra el hecho de que, además de en el epigrama citado, aparece en otros tres casos más en el libro I, si bien sin utilizar expresamente el término 'morir', sino mediante perífrasis, circunloquios o metáforas: Epigr. I 1: uiuenti / post cineres; I 114: ad stygias isset ut umbras / uiuat; I 115: Iam suspendia saeua cogitabas / uiues. 
Un tercer ejemplo de uso en Marcial de antónimos complementarios aparece también en la siguiente agudeza final, en la que se contraponen los términos 'calvo' y 'melenudo'.

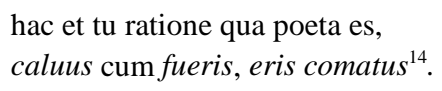

Se trata, de nuevo, de una estructura en quiasmo (adjetivo-verbo sum / verbo sum-adjetivo), con uso de antónimos complementarios aplicados a un mismo verbo sum y colocados los dos antónimos en principio y final de verso. Entre fueris / eris existe, además, una oposición temporal pasado / futuro y una oposición modal subjuntivo/indicativo.

Veamos otro epigrama:

$$
\begin{aligned}
& \text { Cum clamant omnes, loqueris tunc, Naeuole, tantum, } \\
& \text { et te patronum causidicumque putas. } \\
& \text { hac ratione potest nemo non esse disertus } \\
& \text { Ecce, tacent omnes: Naeuole, dic } \text { aliquid }^{15} \text {. }
\end{aligned}
$$

En esta composición aparecen, por un lado, cuatro verbos pertenecientes al mismo campo semántico: clamare, loqui, dicere y tacere ('gritar, hablar, decir y callar'). Por otro lado, para lo que ahora nos interesa, en el último verso - en la punta epigramática - aparecen dos antónimos complementarios: tacere / dicere. Para terminar, entre el primer y el último verso se establece una oposición de modalidad indicativo / imperativo, en estructura quiástica una vez más, y con valor claramente irónico: loqueris... Naeuole / Naeuole dic: "hablas, Névolo / Névolo, habla".

Otras veces, la relación que se establece entre los antónimos no es quiástica, sino paralela:

in steriles nolunt campos iuga ferre iuuenci:

pingue solum lassat, sed iunat ipse labor ${ }^{16}$.

14 Mart., Epigr. I 72. La oposición caluus / comatus reaparece en Epigr. X 83: caluo turpius est nihil comato.

15 Mart., Epigr. I 97. La oposición tacere / dicere reaparece en Epigr. VIII 7; X 101; XII 83. Por su parte, aunque no aparece en la punta epigramática, tal oposición es fundamental en Epigr. V 52. Bajo la forma tacere / loqui la oposición reaparece en Epigr. II 82; VI 41; y, aunque no en la punta epigramática, es fundamental en Epigr. VII 18. Bajo la forma narrare / tacere reaparece en Epigr. XII 35: nam quisquis narrat talia plura tacet.

16 Mart., Epigr. I 107. Esta misma idea antonímica "esterilidad / fecundidad" aparece recogida en la punta epigramática del Epigr. VIII 68, aunque no mediante el uso de antónimos 
En este caso los dos antónimos complementarios ('estéril / fértil') aparecen colocados en el mismo lugar - principio de verso - y siguiendo una estructura paralela: steriles ... campos / pingue solum (adjetivosustantivo / adjetivo-sustantivo). En otro orden de cosas, los verbos lassat y iuиat se relacionan entre sí de forma paradójica: arar los campos fértiles "cansa", pero, sin embargo "agrada". La paradoja reside en el hecho de que el cansancio normalmente, lejos de agradar, molesta o importuna. De ese modo, la paradoja "cansa: agrada" se halla montada sobre una antonimia implícita: en esta ocasión - dirá Marcial - el cansancio, agrada, no desagrada, como hubiera sido lo lógico ${ }^{17}$.

El "paralelismo" en cuanto a la colocación de los antónimos a veces es absoluto. Este es el caso de la siguiente agudeza final:

Issam denique pone cum tabella:
aut utramque putabis esse ueram,
aut utramque putabis esse pictam ${ }^{18}$.

Se trata del conocido epigrama dedicado a la perrita Issa, hecha pintar por su dueño Publio en un cuadro tan conseguido que no era posible - según afirma Marcial de manera hiperbólica - diferenciar la perrita real de la pintada, esto es, la perrita verdadera de la falsa. La oposición que se establece, pues, entre ueram y pictam es una oposición de antónimos "complementarios": 'real, verdadera, natural'/'pintada, falsa, artificial'. Por lo demás, como se ve, salvo el juego de los dos antónimos, el resto de los dos versos finales es idéntico en todo.

El sentido y la función estilística de la repetición es muy claro: Marcial trata de reflejar, formalmente, el parecido casi absoluto existente entre la perrita real y la pintada; y ello lo consigue mediante este artificio formal de la repetición de dos versos casi idénticos.

Hay veces en las que los antónimos "complementarios" aparecen enfrentados entre sí mediante la figura retórica de la antonomasia, lo cual le confiere a la expresión una particular elegancia. Este es el caso de la siguiente punta epigramática:

propiamente dichos: Autumnum sterilis ferre iubetur hiems.

17 Hay que tener en cuenta que la paradoja no es otra cosa, en última instancia, que una antítesis conceptual.

18 Mart., Epigr. I 109. 
nec castrare uelis meos libellos.

Gallo turpius es nihil Priapo $^{19}$.

Marcial se defiende de nuevo en este epigrama de la acusación de obscenidad que algunos le hacían a él y a su obra. El autor replica que la obscenidad es algo consustancial del género epigramático, y que, por tanto, eliminar la obscenidad de su obra sería tanto como pretender que Príapo (caracterizado en la mitología romana con un pene enorme y siempre enhiesto) se convirtiera en un Gallus, es decir, en un sacerdote de Cibeles (sacerdotes que, como es sabido, se castraban en memoria del automutilado Atis, amado de Cibeles). Así pues, Gallo y Priapo - colocados una vez más estratégicamente en principio y final de verso - funcionan claramente aquí como antónimos "por antonomasia" de los conceptos "capado / no capado" o "con pene / sin pene".

Veamos un caso más de uso de antónimos "complementarios":

Quod clamas semper, quod agentibus obstrepis, Aeli, non facis hoc gratis: accipis, ut taceas $^{20}$.

Aquí tenemos, por un lado, la oposición clamare / tacere. Literalmente se trataría de la oposición 'gritar/callar', términos que, en puridad, no son exactamente antónimos, ya que el antónimo propiamente dicho de 'gritar' sería, tal vez, 'susurrar' ('hablar alto/hablar bajo' ${ }^{21}$ ). Sin embargo, en este contexto el verbo tacere significa, por oposición al verbo clamare, no tanto 'callar' como 'dejar de gritar', 'dejar de alborotar'. En efecto, el sentido del epigrama es el siguiente: "Si alborotas siempre, si interrumpes a los abogados durante su actuación, Elio, no lo haces gratis: te dan dinero para que dejes de alborotar". Estamos, pues, propiamente, ante antónimos "complementarios": 'gritar/no gritar', 'alborotar/no alborotar', 'hablar/callar'. Pero además de los antónimos clamareltacere, encontramos todavía en este epigrama otra expresión antonímica: non facis gratis/accipis ('hacer gratis'/"cobrar dinero'). Efectivamente, "hacer gratis" implica "no

19 Mart., Epigr. I 35. Marcial vuelve a hacer uso de esta misma pareja de antónimos en Epigr. XI 72: Drauci Natta sui uocat pipinnam / conlatus cuï Gallus est Priapus.

20 Mart., Epigr. I 95. La oposición clamare/tacere reaparece en Epigr. IX 68, en relación, además - igual que en el presente epigrama -, con la idea de "recibir dinero": Vis, garrule, quantum / accipis ut clames, accipere ut taceas?

21 Se trataría, concretamente, de antónimos "contrarios". 
cobrar dinero", y a la inversa, "no hacer gratis" implica "cobrar dinero"22.

Veamos otro ejemplo de expresión antonímica ${ }^{23}$ de términos "complementarios":

$$
\begin{aligned}
& \text { sed tu ne totiens domini patiare lituras } \\
& \text { neue notet lusus tristis harundo tuos, } \\
& \text { aetherias, lasciue, cupis uolitare per auras } \\
& \quad \text { i, fuge; sed poteras tutior esse } d o m i^{24} \text {. }
\end{aligned}
$$

En primer lugar, encontramos ya una primera oposición de tipo vagamente antitético entre el sustantivo lusus ('chanzas') y el adjetivo tristis ('triste, ceñudo, malhumorado'), términos que remiten a los campos semánticos antitéticos de la alegría y la tristeza, respectivamente. Pero las expresiones más claramente antonímicas se encuentran, como casi siempre, en el último verso: $i$, fuge / domi ("marchar, huir fuera / quedarse en casa, permanecer dentro"). Las oposiciones conceptuales con las que se juega en última instancia son las de "huir" o "quedarse", es decir, las de "huir" o "no huir"; así como las de "fuera" o "dentro". Se trataría en ambos casos de antónimos "complementarios".

Veamos, finalmente, un último ejemplo de este tipo de antónimos:

$$
\begin{aligned}
& \text { Sed tibi non multum est, unum si praesto togatum: } \\
& \text { multum est, hunc unum si mihi, Galle, nego. } \\
& \text { ipse salutabo decuma te saepius hora: } \\
& \text { mane tibi pro me dicet hauere liber }{ }^{25} \text {. }
\end{aligned}
$$

En primer lugar se da en este epigrama una pareja de antónimos complementarios: praesto / nego, 'dar / no dar', 'proporcionar / no proporcionar' ${ }^{26}$

22 La oposición gratis/accipere reaparece en Epigr. XIV 175: Cur a te pretium Danae, regnator Olympi, / accepit, gratis si tibi Leda dedit?. Una oposición similar, gratis/emere, aparece en Epigr. I 29. Otra oposición similar, gratis / numerare, aparece en Epigr. IV 37; XI 62.

23 En este caso no se trata de antónimos puros, sino de expresiones antonímicas, ya que los términos que se oponen ( $i$, fuge / domi) no pertenecen a la misma clase gramatical.

24 Mart., Epigr. I 3.

25 Mart., Epigr. I 108.

26 La oposición praestare/negare es el leitmotiv del Epigr. VII 43: Primum est ut praestes, si quid te, Cinna, rogabo; / illud deinde sequens ut cito, Cinna, neges. / diligo praestantem; non odi, Cinna, negantem: / sed tu nec praestas nec cito, Cinna, negas. Una oposición similar, dare/negare, aparece en Mart., Epigr. II 25; III 54; IV 15; IV 71; VI 10; VI 66. Sobre el campo semántico de la "donación" en Marcial y las "relaciones clasemáticas" del mismo, v. B. García Hernández, art. cit. pp. 241-258. 
(un término es la negación del otro y viceversa). En segundo lugar, aparece una antonimia de expresiones también complementarias, pero llevada a cabo en este caso no mediante procedimientos léxicos, sino mediante procedimientos gramaticales, es decir, mediante la "afirmación / negación" de una misma expresión: non multum est / multum est. En tercer lugar, aparece la oposición decuma ... hora / mane, es decir 'por la tarde/por la mañana'. Por último, es reseñable la oposición antonímica representada por ipse / pro me, que en última instancia remite a la siguiente oposición: "personalmente / no personalmente".

\section{Antónimos "contrarios":}

Veamos, a continuación, el uso que hace Marcial del segundo tipo de antónimos, los denominados antónimos "contrarios", es decir, los que responden a la fórmula siguiente: "La afirmación de A implica necesariamente la negación de $\mathrm{B}$, pero la negación de A no implica necesariamente la afirmación de B". Un primer caso es el siguiente:

$$
\begin{aligned}
& \text { Bellus homo et magnus uis idem, Cotta uideri } \\
& \text { sed qui bellus homo est, Cotta, pusillus homo est }{ }^{27} \text {. }
\end{aligned}
$$

En primer lugar hay en este epigrama una primera oposición conceptual entre "parecer" y "ser", colocadas las dos formas verbales en final de verso: uideri / est. Esta oposición alcanza su verdadero sentido si se tiene en cuenta que el concepto "parecer" lleva implícito en sí mismo el significado de "no ser" (lo que "parece", "no es"), de modo que la oposición "ser / parecer" remite en última instancia a una oposición antonímica más profunda "ser / no ser". Pero, en todo caso, la oposición fundamental en este epigrama es la de los antónimos contrarios magnus / pusillus ('hombre grande / hombre pequeño'). Ahora bien, la agudeza de la composición no consiste en la sola presencia de estos antónimos, sino que la gracia del chiste radica en la triple dilogía presente en los tres adjetivos que intervienen en el epigrama: homo bellus et magnus significa dos cosas al mismo tiempo; por un lado "hombre excelente e ilustre" en el sentido moral o social; por otro lado "hombre guapo y alto o de buena planta" en el sentido físico o estético. Por su parte, el adjetivo pusillus tiene también un doble sentido: en la vertiente

27 Mart., Epigr. I 9. La oposición magnus/pusillus reaparece en III 42; III 62. 
puramente física significa 'pequeño o bajo', en oposición al magnus 'alto o de buena planta'; pero en la vertiente moral o social pusillus significa 'insignificante, bajo o despreciable', en oposición al magnus 'ilustre, excelso, esclarecido'. En el juego entre los seis significados de los tres adjetivos - según se actualice uno u otro sentido - es donde reside la gracia del epigrama, cuya traducción más plausible en castellano tal vez sería la siguiente: "Quieres parecer, Cota, un hombre hermoso y alto, pero el hombre que es hermoso, Cota, es un hombre bajo" 28 .

Esta misma oposición "ilustre / despreciable" vuelve a utilizarla Marcial en otro epigrama del libro I, aunque con diferentes adjetivos:

desperanda tibi est ingentis gloria fati:

non potes hoc, tenuis praeda, sub hoste mori ${ }^{29}$.

La antonimia, que en el epigrama anterior se llevaba a cabo con los adjetivos magnus/pusillus, se lleva a cabo ahora mediante los adjetivos ingens Itenuis: "Abandona la esperanza de la gloria de un ilustre destino. No puedes, presa insignificante, morir a manos de ese enemigo".

Veamos un tercer caso de uso de antónimos contrarios:

Adeone pulchra est? Immo foedius nil est.

Quid ergo in illa petitur et placet? Tussit ${ }^{30}$.

Los adjetivos 'hermoso' y 'feo', lo mismo que los anteriores 'grande' y 'pequeño' (llamados por muchos estudiosos "antónimos graduales”), entrarían dentro de los antónimos "contrarios", es decir, aquellos que consisten en el enfrentamiento de dos conceptos situados en los dos extremos o los dos polos de una misma realidad conceptual, y que se ajustan a la fórmula propuesta: "la afirmación de A implica la negación de B, pero la negación de A no implica la afirmación de B" ${ }^{31}$.

28 Porque para muchos romanos la belleza varonil era signo de afeminamiento. Por lo demás la gracia del epigrama está reforzada - desde el punto de vista formal - por el paralelismo existente en el último verso (bellus homo est / pusillus homo est) con paronomasia entre bellus y pusillus.

29 Mart., Epigr. I 51.

30 Mart., Epigr. I 10.

31 Efectivamente, si algo es hermoso o grande, por fuerza no puede ser feo o pequeño; pero el que algo no sea hermoso o el que algo no sea grande no implica necesariamente que sea feo o pequeño. 
Veamos un cuarto caso de antónimos "contrarios", en esta ocasión repetidos por dos veces:

Nec me, quod tibi sim nouus, recuses:

Omnes hoc ueteres tui fuerunt.

Tu tantum inspice qui nouus paratur

an possit fieri uetus sodalis ${ }^{32}$.

Toda la agudeza del epigrama reside en esta oposición de antónimos: "Y que no me rechaces porque soy nuevo para ti: todos tus viejos amigos lo fueron. Tú mira solamente si el nuevo amigo que adquieres puede convertirse en un viejo camarada".

Veamos un quinto caso de antónimos contrarios:

Scribere me quereris, Velox, epigrammata longa. ipse nihil scribis: tu breuiora facis ${ }^{33}$.

En primer lugar tenemos un oposición de persona: "yo / tú". En segundo lugar, se da una antonimia gramatical del tipo "afirmación / negación": "yo escribo / tú no escribes"34. En tercer lugar, en fin, encontramos una antonimia léxica. En concreto, aparecen los antónimos contrarios "largo / corto". Y todo ello se encuentra aderezado con la estupenda ironía del último verso: "Tú mismo no escribes nada: tú los haces más cortos"; ironía que venía ya anticipada por la utilización del nombre parlante Velox: ¡Y tan veloz!, como que nadie puede ser tan rápido en terminar un epigrama como aquel que ni siquiera empieza a escribirlo.

32 Mart., Epigr. I 54.

33 Mart., Epigr. I 110. La oposición longus / breuis reaparece en Epigr. IX 17; XIV 31. Y sobre el mismo asunto que en el presente epigrama, es decir, sobre la excesiva extensión de los epigramas o de sus versos, la oposición longus / breuis reaparece en Epigr. II 1, II 77 y VI 65. Acerca de este motivo de polémica literaria en Marcial, v. M. Citroni, «Motivi de polemica letteraria negli epigrammi di Marziale», en Dialoghi di Archeologia 2, 1968, pp. 259-301, especialmente pp. 269-70. Sobre este mismo asunto v. también R. Ciocci, «Le durate dell'epigramma in Marziale e nella tradizione . Lettura di Mart. III 58», Annali della Facoltà di Lettere e Filosofia, 18, 1985, pp. 186-200.

34 Se trata, en efecto, de una antonimia "gramatical": no escribir es claramente el antónimo gramatical de escribir. Concretamente, la antonimia gramatical basada en la oposición "afirmación / negación" equivale, en el plano semántico, a la antonimia léxica representada por los antónimos "complementarios". Lo que ocurre es que, a diferencia de la antonimia léxica del tipo hablar / callar, en escribir / no escribir la antonimia no se halla lexicalizada, sólo gramaticalizada. Una prueba de que ello es así es que la antonimia léxica hablar / callar es perfectamente conmutable con la antonimia gramatical hablar / no hablar. 
Veamos un sexto caso de uso de antónimos contrarios:

Tam male cum cenem, cur bene, Flacce, lauer? ${ }^{35}$

Esta misma antonimia "mal / bien" (junto a una oposición temporal "pasado / presente": cantasti / cantas) vuelve a aparecer, tal cual, en otro epigrama del libro I:

Cantasti male, dum fututa es, Aegle.

iam cantas bene; basianda non $\mathrm{es}^{36}$.

Veamos un séptimo caso de uso de antónimos contrarios:

Qui ducis uultus et non legis ista libenter omnibus inuideas, liuide, nemo tibi ${ }^{37}$.

Los términos 'todos' y 'nadie' son claramente antónimos contrarios, por ser los dos polos extremos de una gradación ${ }^{38}$. Pero, además de antonimia, en-contramos en este epigrama la figura retórica denominada "antapódosis", figura consistente en la presentación de dos enunciados contrapuestos que se corresponden y oponen miembro a miembro. En el epigrama de Marcial la antapódosis es la siguiente: "ojalá que tú envidies a todos y nadie te envidie a ti". La punta epigramática se ve, finalmente, adornada por la paranomasia, o parecido fónico, que se establece entre inuide y liuide.

Esta misma antonimia, aunque en la variante omnes / nullus, se da también en la siguiente punta epigramática:

omnis ab infuso numeretur amica Falerno,

et quia nulla uenit, tu mihi, Somne, ueni ${ }^{39}$.

Y otro caso muy parecido a éste es el siguiente:

Nullus in urbe fuit tota qui tangere uellet uxorem gratis, Caeciliane, tuam,

dum licuit: sed nunc positis custodibus ingens turba fututorum est: ingeniosus homo $\mathrm{es}^{40}$.

35 Mart., Epigr. I 59.

36 Mart., Epigr. I 94. La oposición male / bene reaparece en Epigr. X 14 y X 46. Por su parte, la oposición similar malus/bonus aparece en Epigr. VI 82, VII 41, VII 81, XII 6 y XII 80.

37 Mart., Epigr. I 40. La oposición omnes / nemo reaparece en Epigr. XII 80.

38 Los antónimos complementarios, por su parte, habrían sido nadie / alguien, pues en este caso un término es la negación del otro y viceversa.

39 Mart., Epigr. I 71. La oposición omnes/nullus reaparece en Epigr. III 79 y V 23.

40 Mart., Epigr. I 73. 
En esta ocasión no estamos ante una antonimia propiamente dicha, pero la verdad es que se le parece mucho. Ahora la oposición no es "ninguno/todos", como antes, sino "ninguno/muchísimos"; es decir, ahora no estamos ante los dos términos extremos de una gradación, ya que el término extremo que se opone a 'ninguno' es 'todos', no 'muchísimos'. Pero, en última instancia, el sentido perseguido por Marcial es un sentido claramente antonímico: "antes nadie quería tocar a tu mujer / ahora todos la quieren tocar". Con esto entramos en una segunda oposición del epigrama: una oposición de tiempo "pasado/presente": nullus in urbe fuit tota / ingens turba fututorum est. Por último, todavía se podría advertir una tercera oposición conceptual de tipo antonímico entre dum licuit y positis custodibus, pues, en última instancia, la oposición se establece entre "mientras se podía, porque no tenía puestas guardas" y "ahora que no se puede, porque sí tiene puestas guardas"; es decir, existe una oposición tácita de conceptos "complementarios" entre "poder/no poder" o entre "mujer con guardas/mujer sin guardas". La gracia del epigrama se cierra con la ironía final con la que termina la composición: ingeniosus homo es.

Veamos un octavo caso de uso de antónimos contrarios, acompañados en este caso por una pareja de antónimos complementarios:

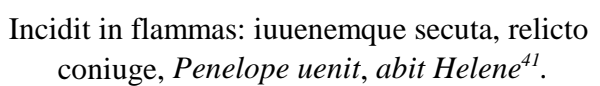

En la agudeza final de este epigrama aparece una pareja de antónimos "contrarios" (uenit / abit) junto a una pareja de antónimos "complementarios por antonomasia", ya que los nombres propios Penelope y Helene están en lugar de los adjetivos 'fiel / infiel', por ser Penélope y Helena el paradigma de la fidelidad y la infidelidad, respectivamente. Y todo ello se encuentra expresado mediante un hermoso quiasmo: los dos antónimos complementarios en los dos extremos, y los dos antónimos contrarios en el centro, dando lugar a la secuencia sustantivo-verbo-verbo-sustantivo: "Penélope vino, marchó Helena".

Veamos un noveno epigrama en el que se juega en todo él con la presencia de antónimos o de expresiones antonímicas contrarias:

Quid nunc saeua fugis placidi lepus ora leonis? frangere tam paruas non didicere feras.

Seruantur magnis isti ceruicibus ungues nec gaudet tenui sanguine tanta sitis.

41 Mart., Epigr. I 62. 
Praeda canum lepus est, uastos non implet hiatus: non timeat Dacus Caesaris arma puer $^{42}$.

En esta composición aparecen, de entrada, tres parejas sucesivas de lo que, a grandes rasgos, pueden ser considerados como antónimos contrarios: saeuus / placidus ('feroz / pacífico'), paruus / magnus ${ }^{43}$ ('pequeño / grande') y tenuis / tantus ('pequeño / tan grande'). Existe, además, una cuarta expresión antonímica implícita en la confrontación que se hace entre el sintagma praeda canum ('presa propia de perros'), es decir, "presa propia de fauces pequeñas", frente a la expresión uastos hiatus ('fauces grandes'). Por lo demás, la liebre y el león, los dos animales protagonistas del epigrama no son más que símbolos - como quedará desvelado al final de la composición de dos conceptos también antonímicos entre sí: "débil / fuerte". En lo que se refiere al contenido general del epigrama, el juego del mismo se basa en la idea de que los débiles (las liebres) no deben tener miedo de los fuertes (los leones), ya que en este caso, paradójicamente, el fuerte no se va a comer al débil. Pues bien, ese mismo tipo de relación 'liebre / león' ("débil / fuerte") es el que Marcial postula para la pareja del verso final: puer / Caesar. El muchacho dacio - dice Marcial - no debe tener miedo del emperador, es decir, el humilde no debe tener miedo del poderoso, y ello porque el poderío de César - dice Marcial, adulando al emperador de turno - nunca dirigirá sus armas contra la debilidad de sus súbditos. Así pues, Caesar / puer remiten en este epigrama, lo mismo que 'león / liebre' a una antonimia metafórica implícita "fuerte / débil" o "poderoso / humilde".

Veamos un caso más de la presencia de varias parejas de antónimos en un mismo epigrama:

Qualem, Flacce, uelim quaeris nolimue puellam? nolo nimis facilem difficilemque nimis.

illud quod medium est atque inter utrumque probamus: nec uolo quod cruciat nec uolo quod satiat ${ }^{44}$.

En primer lugar encontramos una pareja de antónimos complementarios: uelim / nolim ('querer / no querer') ${ }^{45}$. En segundo lugar, una pareja de antó

42 Mart., Epigr. I 22.

43 La oposición magnus/paruus reaparece en Epigr. XI 68 y XIII 78. Y en Epigr. XIV 195: Tantum magna suo debet Verona Catullo, / quantum parua suo Mantua Vergilio.

44 Mart., Epigr. I 57.

45 Se trata de un caso claro de disimetría entre las lenguas latina y española: en latín, la 
nimos contrarios: facilis / difficilis, antónimos expresados mediante un elegante quiasmo: nimis facilem difficilemque nimis, con repetición del adverbio nimis en los dos extremos y con el enfrentamiento en el centro de los dos antónimos ${ }^{46}$; para terminar concluyendo el poeta en el verso tercero que a él le gustan las mujeres que están en medio y entre ambas, es decir, las que no son ni demasiado fáciles ni demasiado difíciles. Y ello se recoge, finalmente, en el cuarto y último verso con una nueva pareja de antónimos contrarios: cruciare / satiare ('atormentar / empalagar'), es decir, algo así como "causar excesivo dolor / causar excesivo placer", expresado en este caso mediante un perfecto paralelismo: nec uolo quod cruciat / nec uolo quod satiat ${ }^{47}$.

Veamos, finalmente, un último ejemplo de antónimos contrarios, en conjunción, una vez más, con antónimos complementarios:

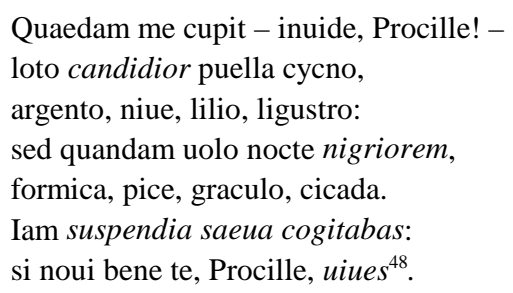

Encontramos en este epigrama, en primer lugar, una oposición de antónimos contrarios en empleo comparativo: candidus/niger $^{49}$. Cada uno de los comparativos lleva tras de sí sendas ristras de cinco ablativos con los que se

antonimia se halla lexicalizada: uolo/nolo; en español, en cambio, esta concreta antonimia no está lexicalizada y por ello nuestra lengua tiene que recurrir al procedimiento gramatical de la "afirmación/negación", que como ya hemos dicho es el equivalente al de los antónimos complementarios: 'querer/no querer'. De todos modos, el latín dispone también de este segundo procedimiento "gramatical", pudiendo sustituir la forma léxica nolo por la forma gramatical nec uolo, como de hecho aparece en el último verso de este mismo epigrama.

46 La oposición facilis/difficilis reaparece en Epigr. XIII 48. Así como en Epigr. XII 46, basado todo él en el uso de la antonimia: Difficilis facilis, iucundus acerbus es idem: / nec tecum possum uiuere nec sine te.

47 Y para rematar toda esta obra de fina ingeniería literaria, obsérvese la paronomasia o parecido fónico que las tres parejas de antónimos mantienen entre si: uelim / nolim, facilem / difficilem, cruciat / satiat.

48 Mart., Epigr. I 115.

49 La oposición candidus / niger reaparece en Epigr. IV 62. En Epigr. IV 2 la oposición se establece entre candidus, albus / niger. 
compara a la muchacha "blanca" o "negra". El epigrama se cierra con una nueva expresión antonímica, en concreto una antonimia de expresiones "complementarias": suspendia saeua cogitabas / uiues, es decir, "querías morir / vivirás" 50 (con oposición temporal, además, del tipo "pasado / futuro").

\section{Antónimos "inversos"}

Hasta aquí hemos visto el uso que hace Marcial de los que hemos denominado "antónimos opuestos". A continuación veremos el uso que Marcial hace del segundo gran grupo de antónimos, los que hemos denominado "antónimos recíprocos". Y dentro de ellos comenzaremos por los "antónimos inversos", es decir, los que responden, según dijimos, a la siguiente fórmula: "La existencia de A implica necesariamente la existencia de B, y la existencia de B implica necesariamente la existencia de A":

\section{Utque tuis longum dominusque puerque fruantur muneribus, tonsum fac cito, sero uirum ${ }^{51}$.}

Tenemos aquí una pareja de antónimos "inversos": dominus / puer (“dueño / siervo' $)^{52}$. Pero a su lado tenemos, para rematar el epigrama, una pareja de antónimos contrarios: cito / sero ('pronto / tarde') $)^{53}$; que siguen una disposición en quiasmo: tonsum fac cito, sero uirum, con los dos acusativos en los extremos y los dos adverbios en el centro. En cuanto a la primera pareja de antónimos ('dueño / siervo'), la volvemos a encontrar en otro epigrama del libro I:

$$
\begin{aligned}
& \text { A seruo scis te genitum blandeque fateris, } \\
& \text { cum dicis dominum, Sosibiane, } \text { patrem }^{54} \text {. }
\end{aligned}
$$

En este epigrama, de imposible traducción, formado por un solo dístico, aparecen dos parejas de antónimos inversos. Por un lado, genitus / pater

50 La oposición uiuere / mori reaparece en Epigr. VI 32; y en Epigr. VII 84: uiuet, Apelleum cum morietur opus. En Epigr. IV 75 la oposición es uita /mors.

51 Mart., Epigr. I 31.

52 Una oposición similar, dominus / famulus, aparece en Epigr. III 21. En II 18 la oposición es rex / seruus; así como en Epigr. II 68: seruom si potes, Ole, non habere, / et regem potes, Ole, non habere.

53 La oposición cito/sero la vuelve utilizar Marcial en Epigr. V 65. Por otro parte, en Epigr. V 67 aparece una oposición parecida: sero / tunc. También el Epigr. VIII 67 está montado sobre la idea antonímica "pronto / tarde".

54 Mart., Epigr. I 81. 
('hijo / padre'). Por otro lado, seruus / dominus ('siervo / dueño') 55 . Pero el chiste auténtico del epigrama reside en la dilogía o doble sentido de uno de estos antónimos: dominus. Sosibiano estaría utilizando el término en su acepción de fórmula de respeto, es decir, con el significado de 'señor'; pero la contraposición de dominus con el término seruus hace que se actualice en la mente del lector el segundo de los significados de dominus, a saber, el de 'amo', con lo cual queda en evidencia el origen servil de Sosibiano, puesto de manifiesto por él mismo cuando llama dominus a su padre.

La oposición "hijo / padre" que acabamos de ver, vuelve a aparecer de nuevo en otro epigrama del libro I:

$$
\begin{aligned}
& \text { condidit hic natae cineres nomenque sacrauit } \\
& \text { quod legis Antullae, dignior ipse legi. } \\
& \text { ad Stygias aequum fuerat pater isset ut umbras: } \\
& \text { quod quia non licuit, uiuat, ut ossa colat }{ }^{56} \text {. }
\end{aligned}
$$

El enfrentamiento de los antónimos inversos 'hija / padre' tiene sentido porque da pie a una oposición conceptual más profunda sobre la que se construye el epigrama: la de que, por ley de vida, la muerte del padre hubiera sido más apropiada - y más justa, añade Marcial - que la muerte de la hija. Y sin embargo la que está muerta es la hija y el que sigue vivo es el padre. La oposición "morir / vivir" aparece de hecho explícita en dos expresiones de nuevo antonímicas: ad stigias isset umbras ("descender a las sombras del Estige", es decir, "morir") frente a uiuat ("vivir") ${ }^{57}$.

Veamos un último caso del uso por parte de Marcial de antónimos inversos:

$$
\text { Non audire, Celer, sed recitare } \text { cupis }^{58} \text {. }
$$

"No deseas oír (mis epigramas), sino recitarlos tú". Se trata de una variante de la antonimia "escuchar / hablar", pues "recitar" no es otra cosa que "hablar", sólo que en voz alta y, normalmente, ante un auditorio. En última instancia estaríamos ante la antonimia representada por el "autor" frente al "re-

55 La oposición dominus/seruus reaparece en V 57.

56 Mart., Epigr. I 114. La oposición natus / pater, mater reaparece en Epigr. VI 38; XI 12.

57 Una última oposición que aparece en el epigrama es la oposición gramatical "activa/pasiva": legere / legi ('leer / ser leído'). La oposición "activa / pasiva” equivale, en el plano gramatical, a lo que en el plano léxico son los antónimos "inversos". Cf. a este respecto B. García Hernández, art. cit. p. 247.

58 Mart., Epigr. I 63. Una oposición similar, audire / dicere, aparece en Epigr. X 101. 
ceptor". De hecho, la punta epigramática consiste, precisamente, en la acusación al plagiario "Veloz" de querer suplantar al verdadero autor.

\section{Antónimos “alternos"}

Pasemos, finalmente, al empleo por parte de Marcial del segundo tipo de antónimos recíprocos: los que hemos denominado "antónimos alternos", y que responden a la siguiente fórmula: "La existencia de A no implica necesariamente la existencia de B, pero la existencia de B sí implica necesariamente la existencia de A". Este tipo de antonimia aparece una sola vez en el libro I:

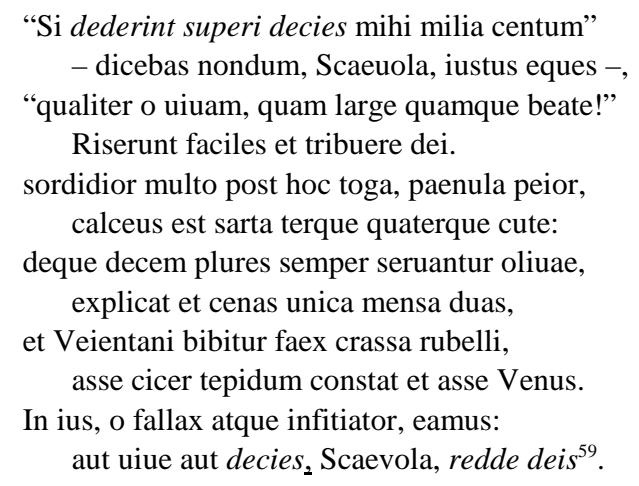

En esta composición la oposición se establece entre el primer y el último verso del epigrama, y se lleva a cabo, concretamente, mediante lo que antes definimos como una antapódosis, es decir, la presentación de dos enunciados contrapuestos que se corresponden y oponen miembro a miembro: si dederint superi decies / decies redde deis ("si los dioses me dieran un millón / devuelve el millón a los dioses"). En lo que se refiere a la antonimia propiamente dicha (dederint/redde) responde, en efecto, a la fórmula que hemos postulado para los antónimos alternos: "dar" no implica “devolver”, pero "devolver" sí implica “dar".

\section{Conclusiones}

Con lo dicho hemos querido ilustrar el uso que hace Marcial de la antonimia léxica, especialmente en la punta epigramática o agudeza final de sus

59 Mart., Epigr. I 103. La oposición dare / reddere reaparece en VI 5; XI 3. Y en V 84: tunc reddam tibi, Galla, quod dedisti. 
epigramas (tanto en los epigramas burlescos como en los epigramas serios). Concretamente, de las 118 composiciones que tiene el libro I de los Epigramas, en 28 de ellas aparece el recurso de la antonimia léxica; y ello representa cerca del $25 \%$ de todo el libro I, lo cual constituye un porcentaje de frecuencia realmente extraordinario. En segundo lugar, hemos querido ilustrar también cómo Marcial reutiliza una y otra vez las mismas o parecidas parejas de antónimos en los sucesivos libros de epigramas que fue escribiendo a lo largo de su vida, de modo que hasta en 71 ocasiones - como hemos ido señalando oportunamente en las notas a pie de página de este trabajo Marcial vuelve a utilizar las mismas o parecidas parejas de antónimos que ya aparecían en el libro I. Ambas cosas ponen de manifiesto un hecho que, en nuestra opinión, hasta ahora no había sido enfatizado suficientemente, a saber, la extrema importancia que alcanza el uso de la antonimia - como procedimiento de estilo y como recurso de agudeza - en la obra epigramática de Marcial. 